Supporting Information of

\title{
Phonon-Assisted Auger Process Enables Ultrafast Charge Transfer in CdSe
}

\section{Quantum Dot/Organic Molecule}

Zhi Wang ${ }^{1,2}$, Mona Rafipoor ${ }^{1,2}$, Pablo García Risueño ${ }^{1,2}$, Jan-Philip Merkl ${ }^{1}$, Peng Han $^{1,3}$, Holger Lange $^{1,2}$ and Gabriel Bester ${ }^{1,2^{*}}$

${ }^{1}$ Institut für Physikalische Chemie, Universität Hamburg, Grindelallee 117, D-20146 Hamburg, Germany.

${ }^{2}$ The Hamburg Centre for Ultrafast Imaging, Luruper Chaussee 149, D-22761 Hamburg, Germany

${ }^{3}$ Department of Physics, Capital Normal University, Beijing Key Lab for Metamaterials and Devices, Beijing 100048, China

*gabriel.bester@uni-hamburg.de

\section{Two QD sizes in experiment.}

We synthesized two sizes of CdSe QD. QD large has an average diameter of $1.8 \mathrm{~nm}$, and it is the one we used to compare with TDDFT simulation. $Q D_{\text {small }}$ has an average diameter of $1.5 \mathrm{~nm}$. We used the same synthesis for both QD samples, except that for $\mathrm{QD}_{\text {small }}$ the time interval between TOPSe and 10 $\mathrm{mL} \mathrm{n}$-hexane (subsequently $10 \mathrm{~mL}$ toluene) injection was $25 \mathrm{~s}$, compared to $40 \mathrm{~s}$ for $\mathrm{QD}$ large. The samples were characterized by UV-vis absorption, collected on Cary 50. A TEM image analysis was conducted using a Jeol JEM-1011 microscope. The results are displayed in Figure S1 and S2.
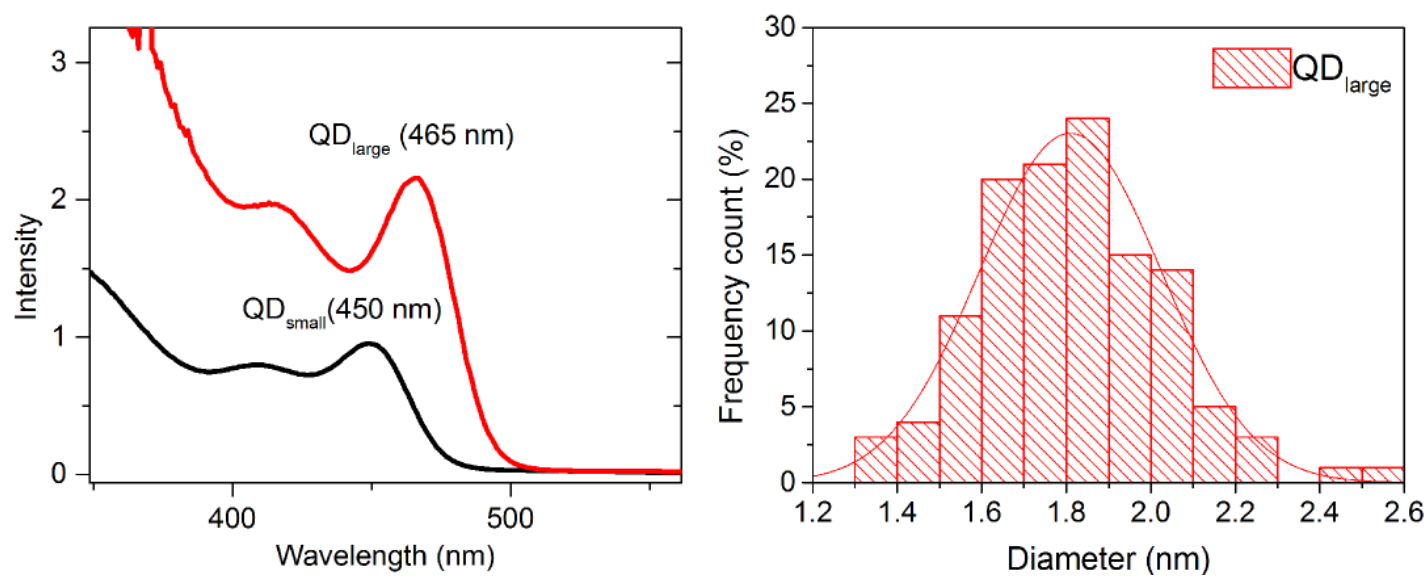

Figure S1 | (Left) the absorption spectra for sample $Q D_{\text {small }}$ (black line) and $Q D_{\text {large }}$ (red line). 
(Right) the diameter distribution of sample $Q D_{\text {large }}$ from transmission electron microscope.
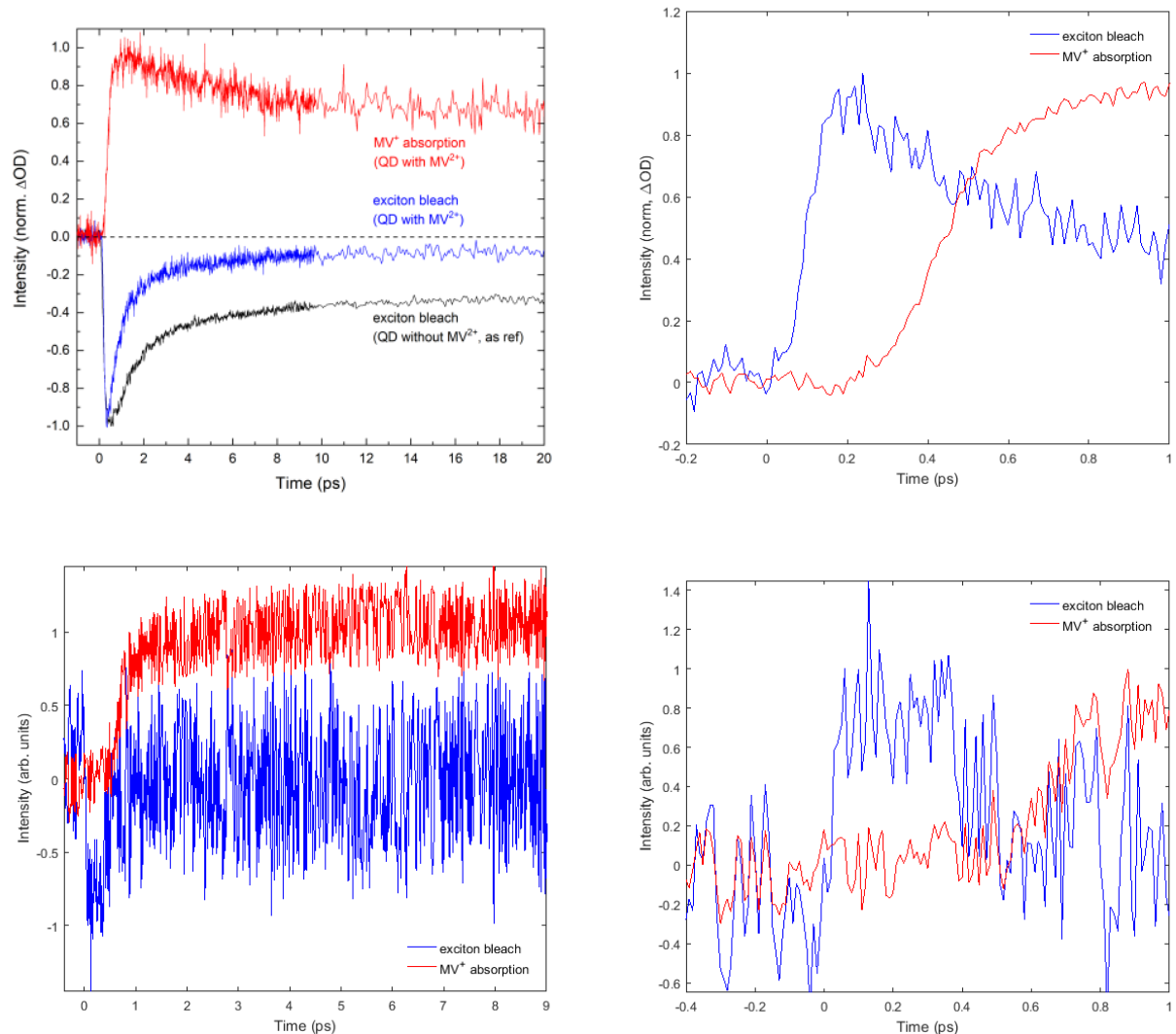

Figure S2 | Upper row: TA spectra for QDlarge with molecule after photoexcitation. Lower row: TA spectra for $\mathrm{QD}_{\text {small }}$ with molecule after photoexcitation. Left column: exciton bleach of $Q D$ (red lines), molecular absorption (blue lines), and exciton bleach of QD without molecule (black lines, as reference) have been normalized. Right column: exciton bleach of QD has been divided by the minimum value while the molecular absorption has been normalized.

II. Band alignment and structural parameters from local density approximation (LDA), generalized gradient approximation (GGA) and GGA + van der Waals (vdW) calculations. 

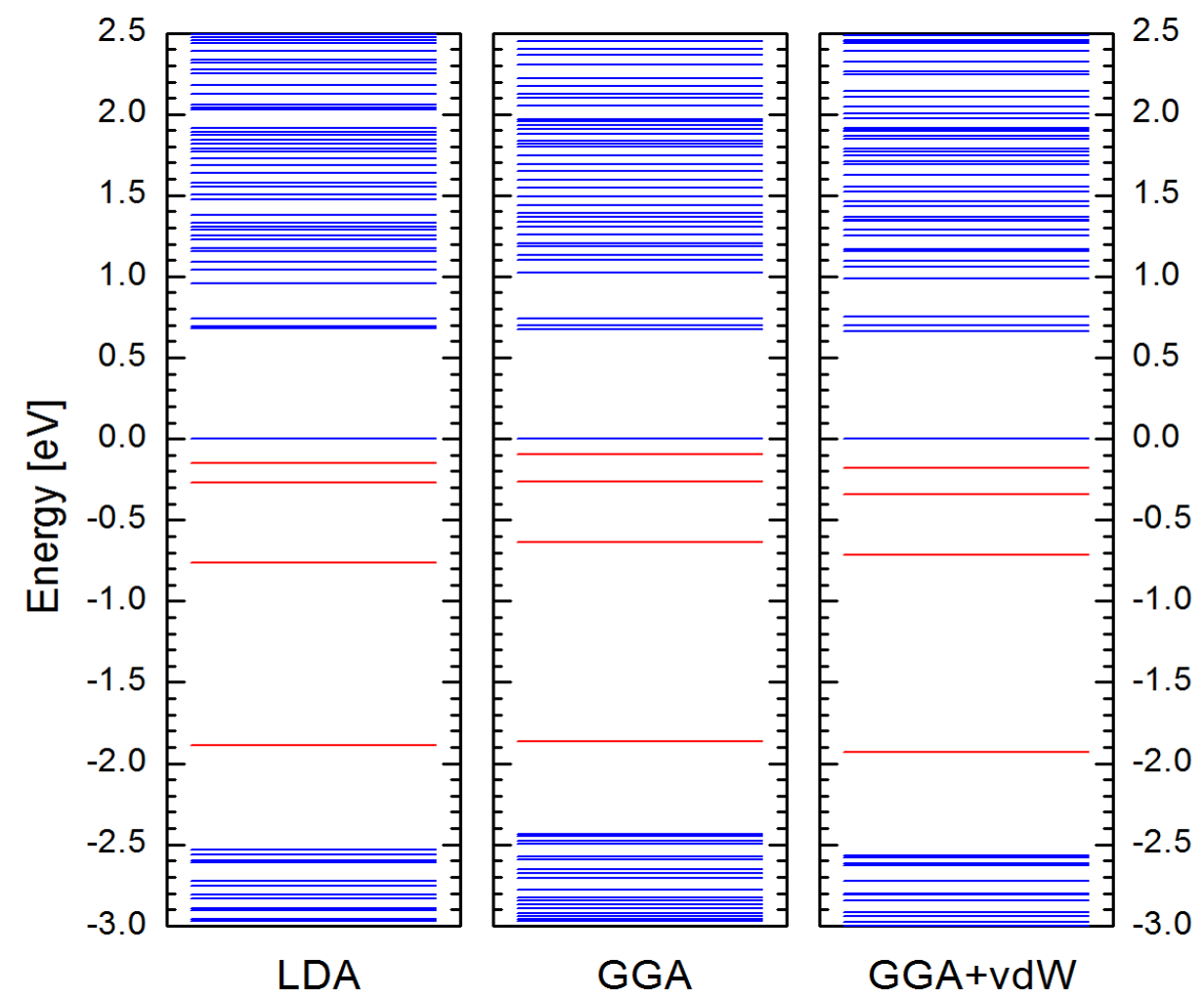

Figure S3 | Band structures from LDA, GGA and GGA + vdW calculations, for the $(\mathrm{CdSe})_{33}$ $(\mathrm{MV}) \mathrm{Cl}_{2}$ system. Blue lines are eigenvalues of QD states, while red lines are ones of molecular states.

Table S1 | Distance between the 2 surface selenide ions and molecule pyridyl $\mathrm{C}(\mathrm{N})$ atoms at the $(\mathrm{CdSe})_{33} /(\mathrm{MV}) \mathrm{Cl}_{2}$ interface, from LDA and GGA+vdW calculations at $0 \mathrm{~K}$.

\begin{tabular}{ccccc}
\hline \multicolumn{1}{c}{ Distance $(\AA)$} & Se-N & Se-C (min) & Se-C (max) & Se-C (avg) \\
\hline \multirow{2}{*}{ LDA } & & & & \\
& 3.272 & 2.667 & 4.141 & 3.466 \\
& 3.474 & 3.348 & 3.642 & 3.498 \\
\hline \multirow{2}{*}{ GGA+vdW } & 3.380 & 2.788 & 4.279 & 3.586 \\
& 3.523 & 3.503 & 3.622 & 3.564 \\
\hline \hline
\end{tabular}

\section{Corrections to DFT energy levels.}

LDA approximation leads to the well-known underestimation of the band gaps. In addition, the $Q D$, the molecule and the non-vacuum solvent environment form a non-uniform dielectric 
environment which affects the electronic states. To tackle this issue, we used a correction scheme, as successfully done previously ${ }^{1,2}$. For the QD-MV-solvent system at each time step $t$, we first decompose the time-dependent LDA single-particle wavefunctions $\varphi_{i}(r, t)$ into two parts using a real space mask function $m(r, t)$,

$$
\varphi_{i}=\varphi_{i} m+\varphi_{i}(1-m)=\varphi_{i}^{\mathrm{QD}}+\varphi_{i}^{\mathrm{MV}}
$$

where

$$
m(r, t)= \begin{cases}1, & \text { if } r \in \mathrm{QD} \\ 0, & \text { if } r \in \mathrm{MV}\end{cases}
$$

Secondly, the many-body corrections on the QD and the molecular conduction band (CB) and valence band (VB) states can be written as,

$$
\begin{gathered}
\Delta_{\mathrm{c}, \mathrm{V}}^{\mathrm{QD}}(t)=\Sigma_{\mathrm{e}, \mathrm{h}}(\mathrm{QD}: \mathrm{QD})+\Sigma_{\mathrm{e}, \mathrm{h}}\left(\mathrm{QD}: \mathrm{MV}^{2+}\right)+\Sigma_{\mathrm{e}, \mathrm{h}}(\mathrm{QD}: \text { sol }), \\
\Delta_{\mathrm{c}, \mathrm{V}}^{\mathrm{MV}}(t)=\Sigma_{\mathrm{e}, \mathrm{h}}\left(\mathrm{MV}^{2+}: \mathrm{QD}\right)+\Sigma_{\mathrm{e}, \mathrm{h}}\left(\mathrm{MV}^{2+}: \mathrm{MV}^{2+}\right)+\Sigma_{\mathrm{e}, \mathrm{h}}\left(\mathrm{MV}^{2+}: \text { sol }\right),
\end{gathered}
$$

$\Sigma_{\mathrm{e}, \mathrm{h}}(a: a)$ is the self-energy correction in compound $a\left(\mathrm{QD}\right.$ or $\mathrm{MV}^{2+}$ ) for unoccupied and occupied state, respectively, and $\Sigma_{\mathrm{e}, \mathrm{h}}(a: b)$ is the polarization energy correction in compound $a$ caused by the dielectric mismatch between $a$ and $b$. Finally, the new Hamiltonian $H^{\prime}$ after correction can be written as,

$$
H^{\prime}(t)=\sum_{i} \sum_{\substack{a, b=\\ \mathrm{QD}, \mathrm{MV}}}\left(\varepsilon_{i}^{L D A}(t)+\Delta_{i}^{a, b}(t)\right)\left|\varphi_{i}^{a}(t)\right\rangle\left\langle\varphi_{i}^{b}(t)\right|
$$

where $\Delta_{i}^{a, b}(t)$ is

$$
\Delta_{\mathrm{c}, \mathrm{V}}^{a, b}=\left\{\begin{array}{c}
\Delta_{\mathrm{c}, \mathrm{V}}^{\mathrm{QD}}, \quad \text { if } a=b=\mathrm{QD} \\
\Delta_{\mathrm{c}, \mathrm{V}}^{\mathrm{MV}}, \quad \text { if } a=b=\mathrm{MV} \\
\left(\Delta_{\mathrm{c}, \mathrm{V}}^{\mathrm{QD}}+\Delta_{\mathrm{c}, \mathrm{V}}^{\mathrm{MV}}\right) / 2, \quad \text { if } a \neq b .
\end{array}\right.
$$

Note that all CB (VB) states have the same correction $\Delta_{\mathrm{C}}^{a, b}\left(\Delta_{\mathrm{V}}^{a, b}\right)$. The corrected wavefunctions $\varphi_{i}{ }^{\prime}(r, t)$ can now be calculated by re-diagonalizing $H^{\prime}(t)$ in the $\left\{\varphi_{i}(r, t)\right\}$ subspace.

$\Sigma_{\mathrm{e}, \mathrm{h}}(\mathrm{QD}: \mathrm{QD})$ and $\Sigma_{\mathrm{e}, \mathrm{h}}\left(\mathrm{MV}^{2+}: \mathrm{MV}^{2+}\right)$ are extract from $\mathrm{GW}$ calculation for bulk CdSe and $\triangle \mathrm{SCF}$ calculation for $\mathrm{MV}^{2+}$ in vacuum, respectively. Notice that the MV molecule is so small that the Coulomb self-energy will be fully screened by the solvent environment.

$$
\begin{gathered}
\Sigma_{\mathrm{e}, \mathrm{h}}(\mathrm{QD}: \mathrm{QD})=\varepsilon_{\mathrm{c}, \mathrm{V}}^{\mathrm{GW}, \text { bulk CdSe}}-\varepsilon_{\mathrm{c}, \mathrm{V}}^{\mathrm{LDA}, \text { bulk CdSe}}, \\
\Sigma_{\mathrm{e}, \mathrm{h}}\left(\mathrm{MV}^{2+}: \mathrm{MV}^{2+}\right)=\frac{\varepsilon_{\mathrm{c}, \mathrm{V}}^{\Delta \mathrm{SCF}, \mathrm{MV}^{2+}}-\varepsilon_{\mathrm{c}, \mathrm{V}}^{\mathrm{LDA}, \mathrm{MV}^{2+}}}{\epsilon_{\text {sol }}},
\end{gathered}
$$

where $\epsilon_{\text {sol }}$ are the optical dielectric constant $\left(\epsilon_{\infty}\right)$ of the solvent. 
$\Sigma_{\mathrm{e}, \mathrm{h}}\left(\mathrm{QD}: \mathrm{MV}^{2+}\right)$ and $\Sigma_{\mathrm{e}, \mathrm{h}}\left(\mathrm{MV}^{2+}: \mathrm{sol}\right)$ has been ignored ( $\left.\equiv 0\right)$ because the $\mathrm{MV}$ molecule is so small that it only has negligible effect on QD energy levels (the band gap of QD in QD-MV ${ }^{2+}$ is only $20 \mathrm{meV}$ smaller than in pure QD), neither forms a dielectrically discontinuous interface between MV and solvent.

Given that the QD has spherical shape and is embedded in an infinitely large solvent environment, $\Sigma_{\mathrm{e}, \mathrm{h}}(\mathrm{QD}: \mathrm{sol}$ ) can be calculated using classical electrostatics equation,

$$
\begin{gathered}
\Sigma_{\mathrm{e}, \mathrm{h}}(\mathrm{QD}: s o l)=\left\langle\varphi_{\mathrm{e}, \mathrm{h}}^{\mathrm{QD}}\left|V_{\mathrm{e}, \mathrm{h}}(\mathrm{QD}: s o l)\right| \varphi_{\mathrm{e}, \mathrm{h}}^{\mathrm{QD}}\right\rangle, \\
V_{\mathrm{e}, \mathrm{h}}(\mathrm{QD}: s o l, r)=\frac{ \pm 1}{2 d \epsilon_{\mathrm{QD}}} \sum_{l=0}^{\infty}\left[(l+1) \frac{\epsilon_{\mathrm{QD}}-\epsilon_{\text {sol }}}{l\left(\epsilon_{\mathrm{QD}}+\epsilon_{\text {sol }}\right)+\epsilon_{\text {sol }}}\left(\frac{d}{R}\right)^{2 l+1}\right],(d<R)
\end{gathered}
$$

where $\epsilon_{\mathrm{QD}}$ and $\epsilon_{\text {sol }}$ are the optical dielectric constant $\left(\epsilon_{\infty}\right)$ of QD and solvent, respectively, $R$ is the radius of QD, and $d$ is the distance from QD center to point $r$. Because the MV molecule is very small, we can write $\Sigma_{\mathrm{e}, \mathrm{h}}(\mathrm{MV}: Q D)$ as

$$
\begin{gathered}
\Sigma_{\mathrm{e}, \mathrm{h}}\left(\mathrm{MV}^{2+}: \mathrm{QD}\right)=V_{\mathrm{e}, \mathrm{h}}\left(\mathrm{MV}^{2+}: \mathrm{QD}\right)\left\langle\varphi_{\mathrm{e}, \mathrm{h}}^{\mathrm{MV}^{2+}} \mid \varphi_{\mathrm{e}, \mathrm{h}}^{\mathrm{MV}^{2+}}\right\rangle, \\
V_{\mathrm{e}, \mathrm{h}}\left(\mathrm{MV}^{2+}: \mathrm{QD}\right)=\frac{\mp 1}{2 d \epsilon_{\mathrm{sol}}} \sum_{l=0}^{\infty}\left[\frac{l\left(\epsilon_{\mathrm{QD}}-\epsilon_{\text {sol }}\right)}{l\left(\epsilon_{\mathrm{QD}}+\epsilon_{\text {sol }}\right)+\epsilon_{\text {sol }}}\left(\frac{R}{d}\right)^{2 l+1}\right],(d>R)
\end{gathered}
$$

where $d$ is the distance from QD center to MV center.

Table S2 | Corrections on LDA energy levels for the 2 QD-MV systems.

\begin{tabular}{ccccc}
\hline \hline \multirow{2}{*}{ Energy level correction $^{*}$} & \multicolumn{2}{c}{$(\mathrm{CdSe})_{33}-\mathrm{MV}^{2+}$} & \multicolumn{2}{c}{ (CdSe) $_{65}-\mathrm{MV}^{2+}$} \\
\cline { 2 - 5 } & $\mathrm{e}(\mathrm{eV})$ & $\mathrm{h}(\mathrm{eV})$ & $\mathrm{e}(\mathrm{eV})$ & $\mathrm{h}(\mathrm{eV})$ \\
\hline$\Sigma_{\mathrm{e}, \mathrm{h}}(\mathrm{QD}: \mathrm{QD})$ & 0.625 & -0.746 & 0.625 & -0.746 \\
\hline$\Sigma_{\mathrm{e}, \mathrm{h}}\left(\mathrm{QD}: \mathrm{MV}^{2+}\right)$ & 0 & 0 & 0 & 0 \\
\hline$\Sigma_{\mathrm{e}, \mathrm{h}}(\mathrm{QD}: \mathrm{sol})$ & 0.194 & -0.231 & 0.173 & -0.207 \\
\hline$\Sigma_{\mathrm{e}, \mathrm{h}}\left(\mathrm{MV}^{2+}: \mathrm{QD}\right)$ & -0.234 & not & -0.151 & not applied \\
\hline$\Sigma_{\mathrm{e}, \mathrm{h}}\left(\mathrm{MV}^{2+}: \mathrm{MV}^{2+}\right)$ & 1.032 & not applied & 1.032 & not applied \\
\hline$\Sigma_{\mathrm{e}, \mathrm{h}}\left(\mathrm{MV}^{2+}:\right.$ sol $)$ & 0 & 0 & 0 & 0 \\
\hline \hline
\end{tabular}

* It only shows the values at $\mathrm{t}=0$.

** Corrections are not applied on $\mathrm{MV}^{2+}$ occupied states because they are very deep in energy ( $\sim 5 \mathrm{eV}$ deeper than QD HOMO). 
Table S3 | Comparison between raw LDA, B3LYP, HSE03, LDA after correction and experimental data on CdSe QD band gap and $\mathrm{MV}^{2+}$ gas phase ionization potential (IP).

\begin{tabular}{|c|c|c|c|c|c|}
\hline & \multicolumn{2}{|c|}{$(\mathrm{CdSe})_{33}$ gap (eV) } & \multicolumn{2}{|c|}{$(\mathrm{CdSe})_{65}$ gap (eV) } & \multirow{2}{*}{$\begin{array}{c}\mathrm{MV}^{2+} \text { gas } \\
\text { phase IP } \\
\text { (V) }\end{array}$} \\
\hline & quasi-particle & optical $^{*}$ & $\begin{array}{l}\text { quasi- } \\
\text { particle }\end{array}$ & optical $^{*}$ & \\
\hline Raw LDA & 2.277 & 1.449 & 1.728 & 1.113 & 10.529 \\
\hline$B 3 L Y P^{3}$ & 3.654 & 2.826 & 3.057 & 2.442 & 11.109 \\
\hline HSEO $^{4}$ & 3.404 & 2.576 & 2.798 & 2.183 & 11.421 \\
\hline $\begin{array}{l}\text { LDA after } \\
\text { correction }\end{array}$ & 4.072 & 3.244 & 3.478 & 2.863 & 12.238 \\
\hline \multirow{4}{*}{ Experiment } & \multirow{4}{*}{ - } & & & $2.666^{\text {this }}$ & \\
\hline & & $2.990^{5}$ & & work, & $11.5^{\sim 12.6}$ \\
\hline & & $3.399^{6}$ & & $2.73^{5}$ & $2^{7}$ \\
\hline & & & & $2.95^{6}$ & \\
\hline
\end{tabular}

* Optical gaps are calculated using the exciton binding energy obtained from configuration interaction calculations.

\section{Thermalization and nuclear vibrational properties.}

The structures of the two $\mathrm{QD} / \mathrm{MV}^{2+}$ systems have been optimized using the Vienna Ab-initio Simulation Package (VASP) ${ }^{8}$ and thermalized at $300 \mathrm{~K}$ (room temperature) prior to the rt-TDDFT calculations. Thermalization was done using Born-Oppenheimer molecular dynamics (BOMD) with Nosé-Hoover thermostat ${ }^{9}{ }^{10}$. During the MD procedure, the mass of the ligand pseudo-hydrogens is set to the oxygen mass (15.999 proton mass) to avoid the potential risk of the unphysical ( $\mathrm{Cd}, \mathrm{Se})-\mathrm{H}$ vibrational modes. After 3 ps thermalization, the $\mathrm{QD} / \mathrm{MV}^{2+}$ systems have reached the thermalequilibrium state. The phonon spectrum extracted from the BOMD (Figure S4) agrees well with previous work $^{11,12}$. 

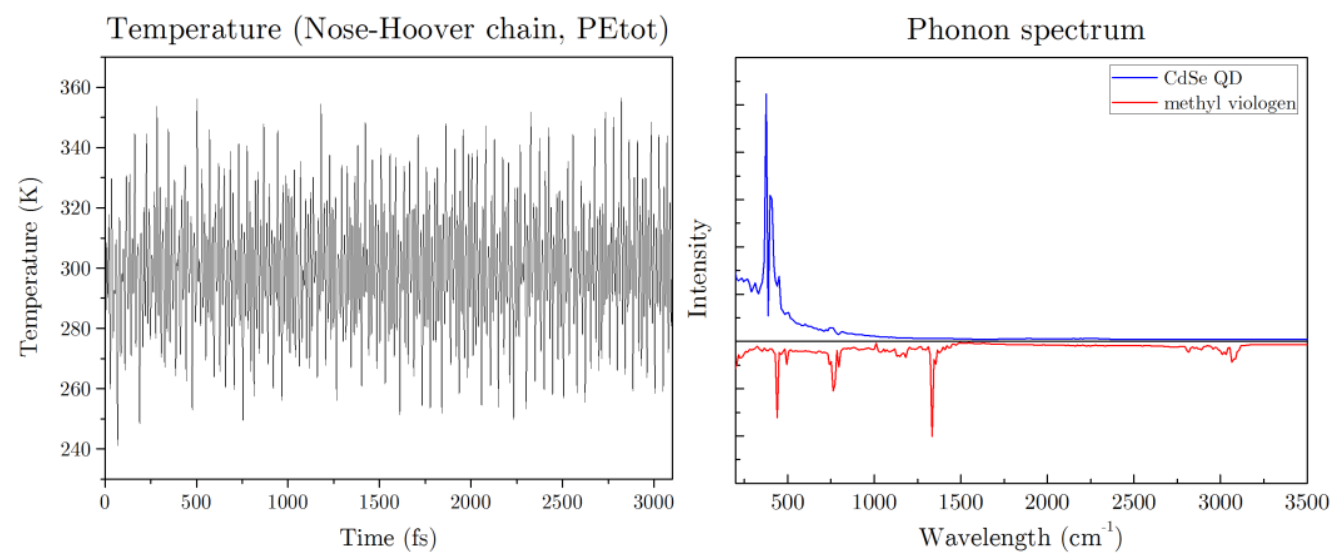

Figure S4 | The thermalization at $300 \mathrm{~K}$ for $(\mathrm{CdSe})_{33}-(\mathrm{MV}) \mathrm{Cl}_{2}$ system. (a) System temperature in 3.1 ps; (b) phonon (vibrational) spectrum extracted from ion motion in (a) using autocorrelation function (ACF) method.

\section{V. rt-TDDFT-NAMD, frozen-ion rt-TDDFT and BOMD procedures.}

Rt-TDDFT calculations are done using the PEtot package ${ }^{13}$. PEtot is a plane wave non-local pseudopotential code designed to tackle large systems ( $10^{3}$ atoms). Its high-speed rt-TDDFT module has been recently developed to extend the capability to non-adiabatic processes in the subpicosecond region. In our system, a set of norm-conserving pseudopotentials has been used with a 50 Ry energy cutoff. LDA (with corrections as shown in Section III above) has been used for the exchange-correlation potential $V_{x c}$.

At $\mathrm{t}=0$, in rt-TDDFT-NAMD and frozen-ion rt-TDDFT calculations, one electron is excited from the QD HOMO to the QD LUMO to mimic the photoexcitation. Subsequently ( $t>0)$, our high-efficient rtTDDFT code is applied to the photoexcited states to deliver the TD electron wavefunctions by solving the TD Schrödinger equation with a time step of $0.05 \mathrm{fs}$ for the electronic system, and let the nuclei move following Newton's law using the ab initio calculated forces (for rt-TDDFT-NAMD) or freezing all nuclei (for frozen-ion rt-TDDFT). For BOMD, there is no initial (photo) excitation.

\section{Vibrational modes and electron-vibrational coupling calculation.}

To find the vibrational frequencies $\omega_{\nu}$ and eigendisplacements (normal modes) $\xi_{\kappa \alpha}^{v}$ we solve the dynamical equation:

$$
\sum_{\kappa \alpha} D_{\kappa \alpha, \kappa^{\prime} \beta} \xi_{\kappa \alpha}^{v}=\omega_{\nu}^{2} \xi_{\kappa^{\prime} \beta^{\prime}}^{v}
$$




$$
D_{\kappa \alpha, \kappa^{\prime} \beta}:=\frac{1}{\sqrt{M_{\kappa} M_{\kappa^{\prime}}}} \frac{\partial^{2} E^{B O}}{\partial R_{\kappa \alpha} \partial R_{\kappa^{\prime} \beta}},
$$

where $\kappa$ and $\kappa^{\prime}$ are the nucleus indices, $\alpha$ and $B$ are the Cartesian coordinate indices, $M_{\kappa}$ is the nuclear mass of atom $\kappa, E^{B O}$ is the BO energy of the system and $v$ is the index of phonon branch. Note that $\xi^{v}$ are vectors of $3 N$ components ( $N$ being the number of atoms) corresponding to the $3 N$ 6 vibrational modes.

We perform the calculation of the vibrational frequencies and normal modes using Quantum Espresso $^{14}$. We use a plane-wave cutoff of $50 \mathrm{Ry}$; the simulation cell is $30 \times 30 \times 30 \AA^{3}$; the exchange correlation functional is $\mathrm{BLYP}^{15,16}$ and we use $\mathrm{HGH}$ norm-conserving pseudopotentials ${ }^{17}$. The total force on the atoms after relaxation is $7.8 \times 10^{-5}$ Ry/a.u.

Treating electron-vibrational interaction as a perturbation $H^{\prime}$ of the ground-state Hamiltonian $H^{0}$ we have

$$
H^{\prime} \equiv \sum_{\kappa a} \frac{\partial H^{0}}{\partial R_{\kappa \alpha}} u_{\kappa \alpha}=\sum_{v} \sum_{i j} g^{v}(i, j)\left(\hat{a}_{v}+\hat{a}_{v}^{\dagger}\right) \hat{c}_{j}^{\dagger} \hat{c}_{i},
$$

where $u_{\kappa \alpha}$ are the nuclear displacements, $i$ and $j$ are the indices of electronic state, $\hat{a}_{v}, \hat{a}_{v}^{\dagger}$ are the phonon annihilation and creation operators and $\hat{c}_{i}, \hat{c}_{i}^{\dagger}$ are the electron annihilation and creation operators; $g^{v}(i, j)$ are the electron-phonon coupling matrix elements, which are given by:

$$
g^{v}(i, j) \equiv \sum_{\kappa \alpha} \sqrt{\frac{\hbar}{2 M_{\kappa} \omega_{v}}} \xi_{\kappa \alpha}^{v}\left\langle j\left|\frac{\partial H^{0}}{\partial R_{\kappa \alpha}}\right| i\right\rangle
$$

and were calculated via finite-difference ${ }^{18}$.

\section{Expansion of the MD trajectories as combination of vibrational modes.}

The TD nuclear positions $\left\{\boldsymbol{R}_{\kappa}(t)\right\}$ are obtained from MD calculations, where $\kappa$ is the atom index. The eigendisplacements for the molecule are given as $\left\{\xi_{\kappa \alpha}^{v}\right\}$ with eigenfrequencies $\omega_{v}$. Before we expand $\left\{\boldsymbol{R}_{\kappa}(t)\right\}$ in terms of $\left\{\xi_{\kappa \alpha}^{v}\right\}$, we need to remove the global drifts and rotations from $\left\{\boldsymbol{R}_{\kappa}(t)\right\}$,

$$
\overline{\boldsymbol{R}}_{\kappa}(t)=S(t) \cdot\left(\boldsymbol{R}_{\kappa}(t)-\boldsymbol{R}_{C}(t)\right)+\boldsymbol{R}_{C}(0),
$$

where

$$
\boldsymbol{R}_{C}(t)=\frac{1}{N} \sum_{\kappa=1}^{N} \boldsymbol{R}_{\kappa}(t), \quad \boldsymbol{R}_{C}(0)=\frac{1}{N} \sum_{\kappa=1}^{N} \boldsymbol{R}_{\kappa}(0),
$$

while the $3 \times 3$ rotation matrix $S(t)$ is obtained from 


$$
M(t)=\boldsymbol{R}_{C}(t) \cdot\left(\boldsymbol{R}_{C}(0)\right)^{T}, \quad M=U \Sigma V^{\dagger}, \quad S=V U^{\dagger},
$$

where the matrices $U, \Sigma$ and $V$ are the singular value decompositions of matrix $M$.

The expansion of $\left\{\overline{\boldsymbol{R}}_{\kappa}(t)\right\}$ as $\left\{\xi_{\kappa \alpha}^{v}\right\}$ can then be written as

$$
a_{v}(t)=\sum_{\kappa \alpha} \xi_{\kappa \alpha}^{v} \cdot \bar{R}_{\kappa \alpha}(t),
$$

where $\alpha$ are the three Cartesian dimensions. Finally, we Fourier transform the coefficients

$$
a_{v}\left(\omega_{k}\right)=\sum_{n=0}^{N-1} a_{v}(t) e^{-\frac{i 2 \pi n k}{N_{t}}}, \quad k=0,1, \ldots, N_{t}-1,
$$

where $N_{t}$ is the total number of time steps, $\Delta t$ is the time interval of the MD calculation, and $\omega_{k}=$ $2 \pi k / N_{t} \Delta t$

VIII. Electron-nuclear vibrational interaction.

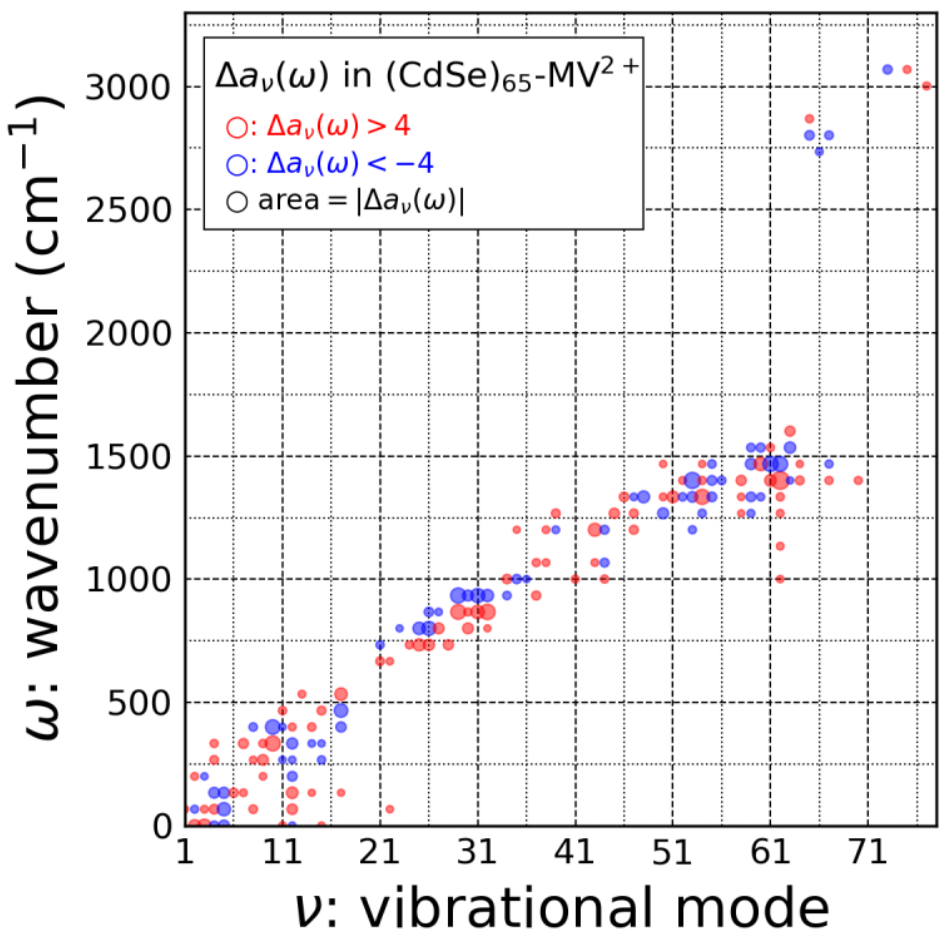

Figure S5 | 2-D vibrational energy redistribution map $\left(\Delta a_{v}(\omega)\right)$ in the first $500 \mathrm{fs}$ of CT process in $(\mathrm{CdSe})_{65}-(\mathrm{MV}) \mathrm{Cl}_{2}$ system.

\section{Prominent molecular vibrational modes in CT process:}


Table S4 | The 8 most prominent molecular modes in CT process.

MV mode \#




(Ring) in-plane C-C-C




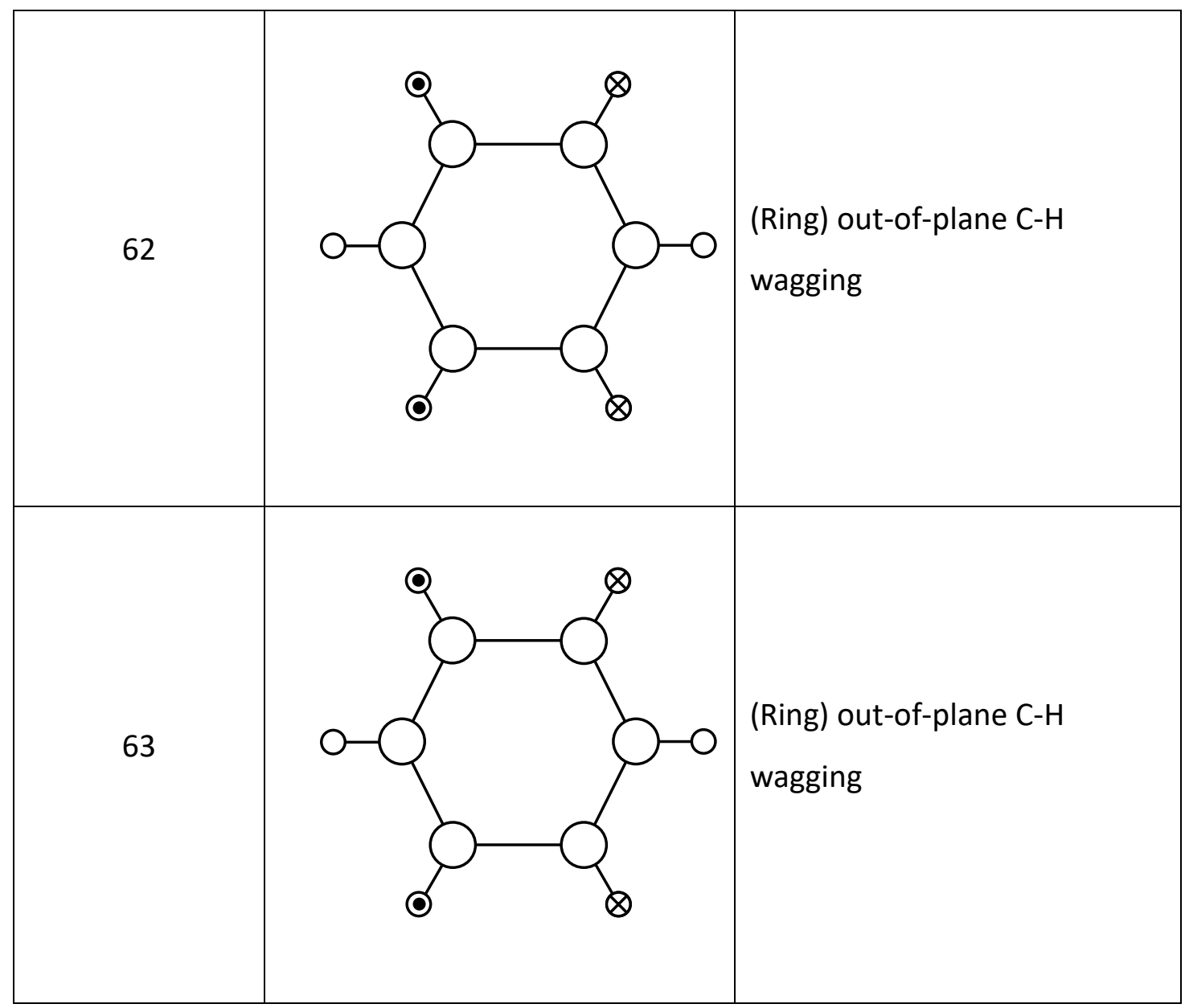

\section{References}

(1) Bester, G. Electronic Excitations in Nanostructures: An Empirical Pseudopotential Based Approach. J. Phys. Condens. Matter 2008, 21, 023202.

(2) Wei, H.; Luo, J.-W.; Li, S.-S.; Wang, L.-W. Revealing the Origin of Fast Electron Transfer in TiO2Based Dye-Sensitized Solar Cells. J. Am. Chem. Soc. 2016, 138, 8165-8174.

(3) Becke, A. D. A New Mixing of Hartree-Fock and Local Density-Functional Theories. J. Chem. Phys. $1993,98,1372-1377$.

(4) Krukau, A. V.; Vydrov, O. A.; Izmaylov, A. F.; Scuseria, G. E. Influence of the Exchange Screening Parameter on the Performance of Screened Hybrid Functionals. J. Chem. Phys. 2006, 125, 224106.

(5) Murray, C.; Norris, D. J.; Bawendi, M. G. Synthesis and Characterization of Nearly Monodisperse CdE (E=Sulfur, Selenium, Tellurium) Semiconductor Nanocrystallites. J. Am. Chem. Soc. 1993, 115, 8706-8715.

(6) Yu, W. W.; Qu, L.; Guo, W.; Peng, X. Experimental Determination of the Extinction Coefficient of 
CdTe, CdSe, and CdS Nanocrystals. Chem. Mater. 2003, 15, 2854-2860.

(7) Peon, J.; Tan, X.; Hoerner, J. D.; Xia, C.; Luk, Y. F.; Kohler, B. Excited State Dynamics of Methyl Viologen. Ultrafast Photoreduction in Methanol and Fluorescence in Acetonitrile. J. Phys. Chem. A 2001, 105, 5768-5777.

(8) Kresse, G.; Furthmüller, J. Efficient Iterative Schemes for Ab Initio Total-Energy Calculations Using a Plane-Wave Basis Set. Phys. Rev. B 1996, 54, 11169-11186.

(9) Nosé, S. A Unified Formulation of the Constant Temperature Molecular Dynamics Methods. J. Chem. Phys. 1984, 81, 511-519.

(10) Hoover, W. G. Canonical Dynamics: Equilibrium Phase-Space Distributions. Phys. Rev. A 1985, 31, 1695-1697.

(11) Tang, X.; Schneider, T.; Buttry, D. A. A Vibrational Spectroscopic Study of the Structure of Electroactive Self-Assembled Monolayers of Viologen Derivatives. Langmuir 1994, 10, 2235-2240.

(12) Han, P.; Bester, G. Insights About the Surface of Colloidal Nanoclusters from Their Vibrational and Thermodynamic Properties. J. Phys. Chem. C 2012, 116, 10790-10795.

(13) Wang, L.-W. PEtot Package. http://cmsn.lbl.gov/html/PEtot/PEtot.html.

(14) Giannozzi, P.; Baroni, S.; Bonini, N.; Calandra, M.; Car, R.; Cavazzoni, C.; Ceresoli, D.; Chiarotti, G. L.; Cococcioni, M.; Dabo, I. QUANTUM ESPRESSO: A Modular and Open-Source Software Project for Quantum Simulations of Materials. J. Phys. Condens. Matter 2009, 21, 395502.

(15) Becke, A. D. Density-Functional Exchange-Energy Approximation with Correct Asymptotic Behavior. Phys. Rev. A 1988, 38, 3098-3100.

(16) Lee, C.; Yang, W.; Parr, R. G. Development of the Colle-Salvetti Correlation-Energy Formula into a Functional of the Electron Density. Phys. Rev. B 1988, 37, 785-789.

(17) Hartwigsen, C.; Gœdecker, S.; Hutter, J. Relativistic Separable Dual-Space Gaussian Pseudopotentials from H to Rn. Phys. Rev. B 1998, 58, 3641-3662.

(18) Han, P.; Bester, G. First-Principles Calculation of the Electron-Phonon Interaction in Semiconductor Nanoclusters. Phys. Rev. B 2012, 85, 235422. 